\title{
Misidentified Flying Objects? An Integrated Psychodynamic Perspective on Near-Death Experiences and UFO Abductions
}

\author{
Stuart W. Twemlow, M.D. \\ Topeka Institute for Psychoanalysis \\ University of Kansas School of Medicine, Wichita
}

\begin{abstract}
This article proposes an integrated psychodynamic perspective to account in part for a variety of similarities between near-death experiences and UFO abductions. The psychodynamic psychology of these experiences implies that their "realness" is mainly a function of that psychology, rather than primarily of an objectifiable external reality. Clinical and research examples highlight the theoretical and practical usefulness of this model.
\end{abstract}

An integrated psychodynamic view of reality grew out of my need to conceptualize the nature of what is "really real," derived from my work over several decades with patients and with students of the martial and meditative arts who had experienced paranormal phenomena that defied neat categorization (Twemlow, Hendren, Gabbard, Jones, and Norris, 1982). Much of the heat generated over controversial topics like these anomalous phenomena depends largely on

Stuart W. Twemlow, M.D., is in the private practice of psychiatry and psychoanalysis and serves on the Faculty of the Topeka Institute for Psychoanalysis, Menninger Foundation, Topeka, KS, and as Clinical Professor of Psychiatry at the University of Kansas School of Medicine, Wichita, KS. He is also Chief Instructor at the School of Martial and Meditative Arts in Topeka, KS, and Vice-President of the United States Kempo Federation. This paper was adapted from his keynote address to the Treatment and Research on Experience Anomalous Trauma (TREAT) Conference, Kansas City, KS, March 1991. Reprint requests should be addressed to Dr. Twemlow at 5040 S.W. 28th Street, Topeka, KS 66614 . 
whether or not they are presented in a controversial and provocative way-a paradox that will become clearer as this model is developed.

The major point of controversy in the field of unidentified flying object (UFO) abductions and near-death experiences (NDEs) has to do with their credibility as "really real" phenomena. Thus it is worth reviewing how this "really realness" is established. Positions on this complex problem can be divided into the following four categories. First, there is the view that these phenomena are totally internal or subjective; that is, they are the products of fantasy, hallucination, delusion, or imagination. Second, there is the view that these phenomena are totally external or objective; that is, they come from other galaxies or dimensions, a form of scientific materialism.

Third, there are the mythic theories, in which the story-weaving quality is the reason in and of itself for the narrative myth and there is little interest in establishing it as an objective phenomenon beyond question. For example, Henri Ellenberger (1970) described a mythopoeic function of the unconscious, a story-weaving capacity implied in the complex Jungian archetypal psychologies.

Fourth, there is an integrated psychodynamic perspective, which bridges and integrates the three previous positions and allows a flexibility not available in these other positions. This model holds that the assumption of an independent and discrete separation between the observer and the observed is a false position leading to misunderstanding and misinterpretation. The "really realness" of this perspective is that reality is always participatory, involving a quasi-empathic step in which the data of experience are of a higher value than so-called objective or "external" data. Objectivity is considered to be largely an artifact of measurement and ideology. Thus knowledge is obtained by this quasi-empathic participatory step and the interface between subject and object is the source of what is defined as knowledge or experience. This knowledge has an inherent value above physical reality, since physical reality is considered to be largely a property of a number of unacknowledged and unprovable assumptions made by positivist scientists.

Before examining the underpinnings of the psychodynamic perspective, it might be instructive to review briefly the perennial "mind/body problem," as summarized in Table 1. It is within the confines of this ongoing debate that issues of what realness is have been defined, obscured, and redefined over the past several thousand years by philosophers and scientists alike. With my humble apologies to philosophers for the oversimplification, this issue boils down to the question of whether mind and brain are one entity, the monist position, or two, the dualist. The mind/ body debate is, in my opinion, a nicely circumscribed arena and model for 
the issue of what "really real" is. In this debate, the issue of whether mind can exist independent of the brain is the central paradox.

An integrated psychodynamic perspective falls in the realm of the structural identity hypotheses of Gordon Globus (1973a, 1973b; Globus and Franklin, 1980), a form of identity thesis (Feigl, 1975). Globus postulated that the fundamental aspects of reality are neither mental nor physical but what he called structural. This "common structure" varies with the position of the observer; the concepts of mental and physical are related to different ways of knowing the common structure depending on one's perspective. A scientist may maintain a

\section{Table 1}

\section{Positions on the Mind/Body Problem}

I. Dualism: two realms exist, (1) matter and (2) spirit in metaphysical terms, or mind in nonmetaphysical terms

A. Interactionism: bodily actions and consciousness affect each other, as posited by Rene Descartes and in the introspectionism of William James

B. Parallelism: brain activity and consciousness are perfectly correlated but do not interact, as posited by Gottfried Wilhelm von Leibnitz

C. Epiphenomenalism: consciousness is an acausal byproduct of brain functioning, as posited by B. F. Skinner

II. Monism: only one realm exists

A. (Scientific) Materialism: only the objective world exists, as in the behaviorism of John B. Watson

B. Idealism: only the subjective world exists, as posited by George Berkeley

C. Identity Hypothesis: body and mind are two inherent aspects of one basic substance or matter, as posited by Herbert Feigl

D. Structural Identity Hypothesis: body and mind are two apparent aspects of one basic structure, depending on the vantage point of the observer, as in the integrated psychodynamic perspective and Gordon Globus' hypothesis.

E. Emergent Evolutionism: brain function and consciousness are emergent properties of the complex nature of the human organism, as posited by Pierre Teilhard de Chardin

F. Metaphysical Idealism: mind is a sixth sense and creates suffering (dukkha) by mind objects (thoughts), as in the "Mind Only" school of Buddhism, which is very similar to the subjective idealism of George Berkeley 
subject/object distinction, since that physical vs. nonphysical perspective is helpful to the conceptual processes and theories he or she uses; while a mystic or meditator in satori might be inclined to dissolve that distinction, including self-awareness. Thus the nature of knowledge varies as a function of the manner of acquaintance with it, a view similar to the participatory reality outlined above.

The question might arise in the mind of the thinker then of how one establishes a hierarchy of values with such a perspective. In other words, how does one distinguish between correct and incorrect solutions to problems, fake or genuine phenomena, etc.? The discriminatory capacity of an integrated psychodynamic perspective depends on unique aspects of the individual circumstances being measured or evaluated. Whether or not this perspective explains the phenomena to the satisfaction of the observer is central, as opposed to the satisfaction of some idealistic, absolute, Platonic concept of the truth. Thus the injection of the subjective into scientific materialism transforms the idea of realness into a flexible and situational concept.

Before any theory explaining reality and defining "really real" is advanced, its basic assumptions should, in my opinion, be outlined. If this were done, there would be less confusion than currently exists in the field; scientific materialism, for example, would have less irrational impact than it does. The conceptual framework of the integrated psychodynamic perspective is captured within the psychoanalytic concept of transference: reality is essentially a transference reality. That is, reality is an integration of what is "out there" or "really real," and what is "in the head," with each impinging on the other and with reality being defined by the degrees of familiarity or unfamiliarity with the sensory perceptual data being experienced. Glen Gabbard and I have defined such a transference reality as comprising four determinants (Gabbard and Twemlow, 1984), all resulting from the interpretive action of the human brain (Polanyi, 1959).

Models have enormous clinical importance because the way the patient presents data will affect the way those data are processed by the clinician. Textbooks on the scientific method often begin with a prefix extolling the similarities between the scientific method and common sense. Science is seen as nothing more than the familiar procedures of common sense highly organized. This is a grossly misleading analogy. Scientific ways of approaching the universe have only occurred in our culture in the past three centuries and, for too many people, make no sense at all. Actually science as it is practiced is nearly always at odds with common sense. Why then is scientific materialism always invoked as the main model by which reality is assessed? 
Alfred North Whitehead only this century explored the fallacy of the "Property of Simple Location" (Yankelovitch and Barrett, 1970). This property establishes that something is "really real" only when it can be located in space. Such a definition excludes all subjective data, as well as many phenomena considered imaginal (Ring, 1989) or altered states of consciousness (Tart, 1972). Thus the basic assumption of scientific materialism automatically is that the subject and the outside worldthe experiencer and the experience - are capable of existing in a spatial relationship to each other, that is, independently of each other. This assumption is not often acknowledged by "hard-nosed" scientists who seem to be modern equivalents of the early Christian martyrs, guardians of all that is "true" and "good." The twentieth-century scientist, for example, makes no more reference to this assumption than practioners of Euclidean geometry ever acknowledged the four assumptions underpinning Euclidean geometry: that alternate angles are equal, that parallel lines meet at infinity, that complementary angles are equal, and that there are 180 degrees in a straight line.

In line with these caveats, the paradigm I am working from has a set of five basic assumptions. First it is assumed that anomalous or usual behavior is possible for all individuals, but there are no individuals who are completely healthy or "normal" nor any that are completely diseased. "Really realness" is thus a matter of judgment.

The second assumption is that cause and effect relationships are not a simple matter. Issues of multiple causation and overdetermination are very important. For example, not only is a single and invariant cause rarely ever found in human experience, but rather multiple sets of causes operate at different points in time, causing a different experience or none at all. Multiple determinants are required for any psychological event; these causes may not be sufficient by themselves, but are often sufficient when acting together. Physical events may be seen to have multiple causes when examined from this perspective.

The third assumption is that all behavior has meaning. Thus, randomness is regarded as a potential function of statistical manipulation and not necessarily part of the natural order of the universe. Fourth, past experience represented in the unconscious mind influences present reactions and thoughts. Thus, an understanding of the past can and does illuminate and help make sense of the present, including socalled "real" phenomena. Consider even, for example, the efficient and final causes according to Aristotle (Ramzy, 1956).

The final assumption regards the state of mind of the observer: this model implies a view of the universe in which the "cause" and "effect" of an experience lie mainly within the head of the experiencer. There 
are four elements involved in the state of mind of the observer. The first element is the observer's belief system. Gabbard and I have written elsewhere: "We are not likely to see that in which we do not believe" (1984, p. 227). For example, the psychoanalyst sees the Oedipus complex everywhere, while the fundamentalist Christian sees the presence of God. These inherent attitudes toward the nature of reality are not often examined by clinicians, nor obviously defined or thought through by observers or experiencers. However, ample work exists to show that the bias of the experimenter influences the outcome of many scientific experiments, even those using animals. In a remarkable experiment, Robert Rosenthal (1977) showed that rats trained by experimenters who believed the rats were bright performed better in mazes than rats they believed were dumb, even though in reality the rats were no different in brightness.

The second element in the state of mind of the observer is the observer's state of consciousness. Much learning is state-dependent, and it is rather difficult to retrieve data unless the state of consciousness in which those data were learned is defined. Bennett Braun described the basic scientific tenets central to state-dependent learning as follows:

The basic tenet of state-dependent learning is that something that is learned in one neuropsychophysiological (NPP) state is most expeditiously retrieved under the same neuropsychophysiological state. If the reinforcement of behavior occurs in sufficiently disparate, dissociated NPP states, the effects of the interaction will not be available under the usual NPP state. If the NPP states are too disparate, retrieval is not possible. $(1988$, p. 5)

Since we are all in different states of consciousness during the day, influenced by our basic rest and activity cycle, use of nicotine, alcohol, over-the-counter remedies, and shifts in attentional focus, it is no wonder that different forms of data are repressed or dissociated from consciousness, causing serious distortions in certain experiences of reality. Individuals who have had a near-death or UFO abduction experience often have a very definite and very firm conviction about the reality of their experiences, which cannot be dismissed as illness. Much knowledge, in fact, is repressed and out of awareness until the individual enters an altered state of consciousness to recapture it. For example, in the psychoanalytic "free association" model, certain repressed aspects of the individual's nature often emerge into consciousness. 
The third element in the state of mind of the observer is the explanatory usefulness of the paradigm. Buddhists speak of "dependent existence," that is, that the realness of an object largely depends on how you see it. For example, a chair is a useful concept for somebody looking for a place to sit when tired, but that same individual may consider a chair to be an arrangement of atoms in space under other circumstances. The paradigm chosen depends upon its situational usefulness. Buddhists call this phenomenon "emptiness." The Buddhist view takes an extreme position, that the form of an object is entirely dependent upon how you wish to use it. This is called "conditioned genesis": all existence is seen to be dependent on the observational paradigm (Rahula, 1974).

The fourth element in the state of mind of the observer is the observer's narcissistic investment in a particular paradigm. This aspect of reality determination is often obvious in clinical settings, and becomes apparent in scientific meetings where discussions often generate more heat than light. Even scientists tend to defend their pet ideas with a fervor far removed from cold, objective assessment. Debates over psychoanalytic theories are particularly good examples of how strong feelings emerge when indefensible ideas are questioned and of how individuals are forced to take sides based on the degree to which their own self-esteem depends on the truth of a theory. A theory backed only by magic and authority brooks no disagreement.

An integrated psychodynamic perspective on NDEs and UFO abductions would require clear and concise acknowledgement of all these philosophical principles and assumptions. It is my hypothesis that phenomena like NDEs and UFO abductions have no meaning considered separate from the experiencer and the psychodynamic determinants of reality as I have described them. Simply put, once the experience is defined according to these precepts or determinants, then a consensus reality can be described in a way that makes sense to discussants.

There are, however, two further complications. Within the consensus reality, certain experiences are not describable or understandable in words. Such translations are potentially verifiable only within the methodological constraints outlined. From another perspective, that of Ken Wilber's "transformative experience" (1981), many NDErs and a few UFO abductees describe life-changing experiences symbolically different and not describable in the often rational terms of previous models. Wilber considers them to be "symbols emerging from a separate ontological ground of consciousness" (1981, p. 37). Sometimes these worlds intersect, and when paradigms clash, chaos can result (Lane, 1984). 
In my clinical experiences with UFO abductees, what is most often unhelpful to the patient are the various belief systems of therapists who judge the experience on scientific materialistic grounds, however neutral and nonjudgmental they wish to be. They tend to convey to the abductee the idea that there is somehow a psychotic core to the experience. A nonrational experience is not necessarily beyond understanding. It merely requires the understander to be conscious and aware of the presuppositions and basic assumptions he or she brings to the experience itself, and to be willing to use nontraditional conceptual models to describe the experience. Having outlined a paradigm I consider useful in conceptualizing NDEs and UFO abductions, I will now apply the model to try to expand and enrich our understanding of these experiences.

\section{Social and Psychological Characteristics of NDEs and UFO Abductions}

Whatever else NDErs and UFO abductees are, they certainly represent a wide variety of educational backgrounds, levels of psychopathology, ages, and religions (Bullard, 1987). In general, abductees seem to be ordinary individuals, certainly not falling into a characteristic psychopathology category. Gabbard, Fowler Jones, and I found many similarities between NDErs and individuals who reported out-of-body experiences (Gabbard and Twemlow, 1984; Jones, Gabbard, and Twemlow, 1982).

Recent surveys of psychiatric illness in abductees (Bartholomew, Basterfield, and Howard, 1991) showed no circumscribed psychopathology, but did show a proneness to fantasizing. Susan Powers (1991), however, noted that in many surveys, about 20 to 35 percent of fantasy-prone individuals are maladjusted and report a common experience of harsh childhood punishment and abuse. Powers also noted that fantasy-prone individuals use two dominant psychological coping mechanisms: amnesia, to block a painful memory, and creation of a nonordinary reality, including beings such as "guardian angels or extraterrestrials" (1991, p. 48).

Such fantasizers are often highly hypnotizable and report difficulty in discriminating between memories of fantasies and memories of events (Wilson and Barber, 1983). Reports of amnesia are frequently present in anecdotal accounts of extraterrestrial abductions (Hopkins, 1981). George Ganaway pointed out that "UFO abductees are highly hypnotizable, highly imaginative, and typically uncover their first 
memories of an abduction experience during hypnotic interrogation by self-proclaimed UFO abduction experts" (1989, p. 213).

There also seem to be similarities between NDErs and UFO abductees: Kenneth Ring and Christopher Rosing (1990), in a study of the NDE-prone personality, showed that such individuals have a higher incidence of childhood abuse and trauma. In my own study of 339 NDErs and out-of-body experiencers, I found that 32 percent reported having seen a UFO (Twemlow, 1989), compared to 14 percent of the general population as reported in a Gallup Poll (Gallup and Newport, 1991) and 7 percent in a larger random sample of 5,947 American adults conducted by the Roper Organization (Hopkins, Jacobs, and Westrum, 1992). There have also been reports of personality and demographic similarities between NDErs and UFO abductees (Bullard, 1987).

Given these generally agreed upon findings, I will make three assumptions. First, a majority of abductees are not liars nor malingerers, nor severely psychiatrically disturbed-although no doubt some are. Second, the abduction experience, whether or not it can be shown objectively to be "really real," is certainly real to the abductees. It is certainly considered more real than a dream or fantasy, just as are NDEs.

David Lane (1984) wrote that the feeling of certainty is a complex result of sensory input and the interpretive action of the brain, but that ultimately that interpretive action is immeasurable and undefinable. Lane called this the "Chandian effect," after his guru. Subjects in general are angered by any suggestion that the experience was a product of fantasy or psychiatric illness, and there is certainty about the experience as implied by Lane's "Chandian effect"-a certainty of the fact, based on the self-confirming nature of the experience itself.

My third assumption is that the experience is usually reported as highly ego dystonic, and it often provokes a mixture of fear and excitement in the experiencer. Phenomenologically, this reaction of uncanniness is precipitated by a situation in which something occurs that is quite different from what was anticipated. Sigmund Freud (1919) considered this reaction of uncanniness to be related to repressed Oedipal castration anxieties.

UFO abductees handle the experience in various ways. Less than 2 percent-only 6 individuals in one study of more than 300 experiencers-transform the experience into a life-changing positive spiritual experience (Bullard, 1987). Others repress the experience completely. For example, Rima Laibow (1989) reported a 36-year-old professional man who had always felt that his mother had sexually 
abused him until, in working with Laibow, he uncovered possible evidence that enabled him to recast the sexual abuse in the light of UFO abduction. The suggestion that he might have been abducted rather than sexually abused was precipitated by a strong emotional reaction to reading an account of someone else's UFO abduction.

This case is an excellent one for the application of an integrated psychodynamic perspective. For example, if the therapist's belief system and narcissistically invested paradigm had been psychoanalytic, the interpretation given would most likely have been that the concept of the abuser being an alien is far more acceptable to the individual than the idea that his mother may have abused him. If the therapist even unwittingly and with the very best of intentions responded with more interest to the idea that aliens rather than family members have perpetrated this abuse, then the abduction explanation would likely be more acceptable to the patient, though not necessarily more therapeutic in the long term. The abuse would then become further disguised and displaced from reality, and the individual would become more neurotic rather than less. In that instance the therapist's narcissistically invested belief system involving a UFO abduction explanation would collude with the patient's unconscious wishes.

Conscious recollections and vivid dreams or memories can also obscure less phantasmagoric but infinitely more unacceptable thoughts, such as repressed abuse and neglect by parents whom the child loves and depends upon. No encounter with an alien can match the soulmurdering horror of being betrayed by those whom one loves the most. It is not uncommon clinical experience that transference objects become monstrous. For example, in cases of violent and unexpected physical attack on individuals who have been victims of childhood sexual and physical abuse, the attacker might be imbued with characteristics that he or she does not possess, such as monstrous size and ferocious intensity.

It is often difficult for victims to identify their attackers because of these projections of monstrous and alien features. In a recent clinical case, after intensive psychotherapy, an individual who had had an abortion as a teenager described her doctor in monstrous terms; he appeared in a dream as an alien monster with glowing eyes and deformed, dwarf-like body. Parents, especially in cases of incest, are also commonly depicted in dreams as monstrous beings of an alien nature.

Still other abductees convert their experience into a psychiatric illness, usually classified as an acute post-traumatic stress disorder (PTSD). The characteristics of PTSD in UFO abductees appear to be 
little different clinically from those that occur following any severe external trauma.

A number of clinicians have commented that external trauma is a requirement for the post-traumatic response, and that PTSD does not follow traumatic internal phenomena such as in delusional or hallucinatory states or fantasies. Although this is plausible for psychiatric patients who can discriminate reality from fantasy, work with psychotic and borderline patients indicates that internal fantasies subject to the defense of projective identification can be experienced as external trauma, and can produce a post-traumatic-like response in some cases, such as spirit possession and exorcism. In a recently published study of 36 patients recovering from an acute psychotic episode, 30 to 50 percent were found to have PTSD symptoms between 4 and 11 months later (McGorry, Chanen, McCarthy, Van Riel, McKenzie, and Singh, 1991).

Finally, some abductees simply dismiss the experience. Although this response seems unlikely, it does occur (R. Laibow, personal communication, 1991).

\section{Phenomenology of the Experience}

The most striking feature of NDEs and UFO abductions is that the basic descriptive characteristics of the experience are relatively constant among subjects. As Thomas Bullard (1987) pointed out, the pattern could be a lot more imaginatively described if the abductee cared to "spin a story." In my work with near-death and out-of-body phenomena, I have been impressed by the importance of knowing the individual's state of consciousness at the time of the experience and the preexisting general environmental and personal conditions, in order to understand their influence in precipitating the experience and in the way it is processed (Twemlow, Gabbard, and Jones, 1982). Anecdotal accounts tend to imply that the UFO abductees were not expecting the abduction, although I suspect that more intensive studies with less of an advocacy position might show that at the time abductees have been experiencing unusual personal changes in their lives, as were onethird of our NDErs and out-of-body experience subjects (Gabbard and Twemlow, 1984).

The abductee is then usually physically or sexually examined without consent, an important point I will return to later. There is usually a conference or discussion among the aliens, sometimes a tour of the UFO, and occasionally an otherworldly journey. In a small percentage of cases, a transforming religious experience occurs in which the abductee receives a message from a divine being, followed by a return to 
earth. The experience is usually so foreign to the individual that he or she may experience dissociative shifts in time and levels of alertness, with depersonalization and derealization. This basic abduction experience pattern is consensually validated across fairly large numbers of subjects (Bullard, 1987).

Up to this point, my work with NDErs and UFO abductees is quite parallel: both experiences are felt to be more real than a dream and have a characteristic phenomenological pattern, with entry into an unusual, often lighted, area, contact with entities, a border (the spaceship), and a return to earth with significant aftereffects. A major difference is that in 95 percent of NDErs the experience is largely positive, and in 95 percent of UFO abductees, it is mainly negative.

\section{The Affective Reaction}

Both types of experience are more real than a dream, are intensely vivid, and involve the experience of externality, but they provoke vastly different affective reactions. Bullard (1987) described 6 cases of positive affect in his study of more than 300 UFO abductees, although he gave few details. In an NDE reported by Harvey Irwin and Barbara Bramwell (1988), the experience started as a typical positive one but then spontaneously became highly negative and hellish. As Irwin and Bramwell pointed out, in the negative NDE, "beings" are often experienced as severely judgmental, while in the positive NDE, unconditional acceptance and love are often reported from "beings of light." In Irwin and Bramwell's case, however, the NDEr concluded the experience with a more spiritual orientation and a less materialistic view of life. In other words, the overall outcome was positive even though the experience was generally traumatic.

In a recent review, Gabbard and I (1991) concluded that NDEs do not represent so much an experience of imminent physical death, but are what Wilfred Bion (1963) called experiences of catastrophe: a nameless dread or sense of panic as if one's sense of self and one's sense of being something are in danger of being destroyed. Bion believed that this sense of catastrophe remains at the core of existence through life, and that the only appropriate response to it is a "keeping on going in spite of" attitude. The positive NDE can be understood as one manifestation of such faith, and the NDE both as a manifestation and a catalyst for the development of that faith, akin to Ernst Kris's (1975) regression in the service of the ego.

We concluded that NDEs are not necessarily associated with death at all, but with situations that may remind the individual of a sense of 
catastrophe. Why do some individuals evoke faith and have a positive outcome, whereas others do not? The usually negative response of the UFO abductee has to do, in my opinion, with a sudden, less adaptive regression. The UFO abduction is experienced as much more alien or ego dystonic than the near-death experience; the NDE fits much more closely with sociological and cultural mores and religious values than does the abduction experience. Most religions have some concept of salvation or at least a change in a positive direction after death. UFO abductees are much more likely to remember their experiences as abusive or judgmental, and thus to invoke more primitive defenses.

Ilsa Bick (1989) reviewed the appearance of alien children in science fiction and science fantasy movies and publications. Modern children are strongly influenced by science fiction that features extraterrestrial life. A hypothesis that could be tested over time is that UFO abductions will become less terrifying as the culture accepts more and more of these extraordinary and anomalous realities, so that less primitive defenses against them need to be invoked.

\section{The Role of Projective Identification}

Increasingly recognized in psychoanalytic thinking, projective identification involves the projection of unacceptable thought contents into an external container. Many of these projected thoughts are felt not as abstract ideas by the container, but as a sense of invasion by a foreign body in one's body/mind; and thus the individual is affected by the projections. UFO abductees do appear to make regular use of projection on psychological testing in a small series of reported cases (Bloecher, Clamar, and Hopkins, 1985). Such mechanisms have also been used as explanations for spirit possession.

When the container is not a human being, as in the case of UFO abductions, a more complex explanation needs to be invoked. Here Bion's (1957) concept of bizarre objects, derived from his analysis of schizophrenic patients, may be useful. Under the pressure of psychotic illness, fragmented thoughts, sensations, and percepts may be projected onto inanimate external objects that in turn become infused with significant meaning and even life, even though these "bizarre objects" are inanimate. Later research has demonstrated this process of fragmentation and projection in normally functioning individuals, in addition to schizophrenic patients. Thus the meaning attached to a nonhuman or alien container is derived from the "bizarre objects" projected onto it. 
Since those projections are threatening, the individual maintains an identification with the container of his or her projections, as a way of making sure that these projections do not reinvade unexpectedly. In the clinical situation, the therapist tries to transform the projections so they can be safely reintrojected by the patient. In the case of the untreated UFO abductee, the projections remain external, sometimes giving rise to paranoid explanatory fantasies, often involving plots by a criminal underworld or secret service agencies. One such UFO abductee advanced the hypothesis that all adopted individuals were aliens in disguise.

Why then the primitive defenses? Both the NDE defense, a form of adaptive regression, and the UFO abduction defense of pathologic projective identification, have the same function: they are unconscious attempts to reduce anxiety. However, the UFO abduction experience is a far less successful attempt; although the terrifying alien experiences are successfully projected, the individual is trapped in a traumatic scenario by his or her continuing identification with the phenomenon itself, including fears of reabduction, that is, reinvasion by unmodified introjects.

This model illustrates an integrated psychodynamic perspective on NDEs and UFO abductions. Of course, since this is primarily a "mental" model, it emphasizes the quasi-empathic participatory role of the observer and the observed in defining "really realness," with the implication of a continuing modification of experience by this ongoing interpersonal transaction. Thus, whether something is "outside" or "inside" becomes unimportant from the point of view of evidence, and certainly from the point of view of experience; those would simply be levels of intensity or vividness of reality. It seems obvious from the foregoing model of NDEs and UFO abductions that from an integrated psychodynamic perspective, prior history, including childhood events, influences vastly the nature of the experience itself; but many factors present at the time of the experience are also involved in its creation.

All individuals have the propensity for unusual and even pathological experience; all experiences are state-dependent and thus affected by the individual's state of consciousness. The neurological, physiological, and psychological concomitants of arousal and attention have a complex effect on cognition and perception. The integrated psychodynamic view is neither outrageous nor reductionistic; but it sometimes provokes such accusations from individuals who feel the only correct paradigm is that of scientific materialism, and that the experience is thus totally external and independent of the observer. 
Unexpected support for an integrated psychodynamic perspective has come from the work of Daniel Stern (1985), whose studies of psychological development have shown that infants tend to organize what is going on in reality and to construct working models to explain such a reality from a very early age, although they subsequently experience distress if reality fails to match their models. As they grow older, children tend not only to explain the reality they experience, but also to construct reality to organize events and interactions.

In a recent comprehensive book summarizing firsthand accounts of UFO abductions, David Jacobs (1992) reviewed a variety of explanatory theories, with obvious bias. He dismissed various explanations because, according to him, they failed to account for the similarity of phenomenological detail and the extraordinary "convergence of abductee narrative across all cultural boundaries" (p. 302). Jacobs' argument does not hold up to closer examination: human beings all have basic similar mental mechanisms and especially similar basic defenses. It seems highly likely that such intensive psychological experiences tend also to be rather similar, since many of these primitive anxieties had their template laid down very early in nurturing experiences that do not vary greatly between cultures. Jacobs' statement that there is lack of strong personal content in abduction accounts is simply false: clinical experience with abductees in intensive psychotherapy shows that the nature of the experience is very highly colored by past experience.

\section{Conclusion}

There are a number of clinical and research questions raised by this integrated psychodynamic perspective.

\section{Clinical Implications}

Psychiatry is currently again embroiled in a controversy about the importance of real versus subjective experience, due to the resurgence of interest in childhood sexual abuse. How one determines what is "really real" and whether that is important are subjects of an enormous amount of discussion and research. One matter, however, seems to be agreed upon: to be helpful, the therapist must accept the patient's view of reality in a way that is nonjudgmental and neutral. A famous pioneer of psychoanalysis, Sandor Ferenczi, commented in 1932: 
If this is not done with a total belief in the reality of the process, the "shaking up" will lack persuasiveness as well as effectiveness; but if the analyst does have that conviction and the related sympathy for the suffering being, he may, by judicious questioning, which compels the sufferer to think, succeed in directing this being's reflective powers and orientation to the point where it can say and remember something about the circumstances of his shock. (DuPont, 1988, p. 9)

What better advice for someone helping a UFO abductee? We know that neither advocacy nor skepticism will help. Clinically, if the therapist raises questions about the credibility of the abductee, these questions often are a sign of the therapist's countertransference anxiety upon hearing about such experiences.

\section{Research Questions}

A number of questions arise: Why is this phenomenon a relatively modern one, as Bullard (1987) suggested? Is it possibly influenced by modern scientific discoveries or fiction and the media? Why has there not been clear, unequivocal evidence of extraterrestrial contact with our culture by now? It has been reported that there is a statistical likelihood of at least 10,000 inhabited planets in our galaxy (Drake, 1981); why haven't we seen more direct evidence, especially in the light of this veritable modern epidemic of UFO abductions (Bullard, 1987)? Why the mystery and subterfuge? The aliens do not seem to have any special wish to take over or destroy the planet-or at least have not done so yet!

An integrated psychodynamic approach to such questions would postulate that using the model of scientific materialism to test the credibility of such experiences actually has a dampening effect on possible understanding. It may well be that our future "communication," if any, with such "beings" will depend on our adopting more flexible conceptual models for such communication. Perhaps a journey into inner space is required!

The unifying theory of mind and matter proposed by Robert Shacklett and William Gough (1991) contains some interesting speculations that may enable rapprochement between objectivists and subjectivists. These researchers, using a paradigm based on the mathematical model of Roger Penrose's twistor, argued that space/time is not an impermeable barrier confining human experience within the world of matter. They speculated instead that a number of human experiences, including those called "paranormal," can be mediated through a mind operat- 
ing in the abstract spaces of modern quantum mechanics beyond the level of subatomic particles.

They suggested that the human mind contains patterns suggesting knowledge of such spaceless and timeless areas as, for example, Carl Jung's archetypes, and that access to such cognitive and perceptual mechanisms does not follow the natural laws of the space/time bounded by mind.

As should be obvious from my model outlined above, I prefer earthbound-but not pathologizing nor empirically limitingexplanations for NDEs and UFO abductions. I have never fully understood why individuals need to search elsewhere for experiences of awesomeness and wonder, whether it be into the astral planes, other galaxies, or into heaven and hell. In my work as a clinician in both psychoanalysis and general medicine, and in my experiences as a martial artist and practitioner of Zen, I am constantly rediscovering the awesomeness of the human experience itself.

I have no doubt that the human brain is unlimited in its potential and might even be able to create physical reality itself, as Shacklett and Gough (1991) implied. It might be capable of explaining most of the phenomena in our consensually validated reality without extravagant appeal to other "separate" worlds and entities. In my view, humanity's effort to live creatively with the absolute certainty of our own demise and our ability to evolve explanatory paradigms to transcend the human condition are sufficiently awe-inspiring. The model I propose here provides a possible bridge between "subjective" and "objective" realities that I hope will stimulate rapprochement rather than conflict between competing scientific and nonscientific explanatory models.

\section{References}

Bartholomew, R. E., Basterfield, K., and Howard, G. S. (1991). UFO abductees and contactees: Psychopathology or fantasy proneness? Professional Psychology, 22, 215222.

Bick, I. J. (1989). Aliens among us: A representation of children in science fiction. Journal of the American Psychoanalytic Association, 37, 737-759.

Bion, W. R. (1957). Differentiation of the psychotic from the non-psychotic personalities. In W. R. Bion, Second thoughts: Selected papers on psycho-analysis (pp. 43-64). New York, NY: Jason Aronson.

Bion, W. R. (1963). Elements of psycho-analysis. London, England: Heinemann.

Bloecher, T., Clamar, A., and Hopkins, B. (1985). Final report on the psychological testing of UFO "abductees." Mt. Rainier, MD: Fund for UFO Research.

Braun, B. (1988). The BASK model of dissociation. Dissociation, 1, 4-19. 
Bullard, T. E. (1987). On stolen time: A summary of a comparative study of the UFO abduction mystery in true UFOs. Fantasy or reality? In B. Maccabee, (Ed.), A UFO primer. Mt. Rainier, MD: Fund for UFO Research.

Drake, F. D. (1981). Intelligent life in the universe. In G. O. Abell and B. Singer (Eds.), Science and the paranormal (pp. 329-348). New York, NY: Charles Scribner.

DuPont, J. (1988). The clinical diary of Sandor Ferenczi. Cambridge, MA: Harvard University Press.

Ellenberger, H. (1970). The discovery of the unconscious: The history and evolution of dynamic psychiatry. New York, NY: Basic Books.

Feigl, H. (1975). Some crucial issues of mind-body monism. In C. Cheng (Ed.), Philosophical aspects of the mind/body problem (pp. 22-34). Honolulu, HI: University Press of Hawaii.

Freud, S. (1955). The uncanny. In J. Strachey (Ed.), The standard edition of the complete psychological works of Sigmund Freud (Vol. 17, pp. 217-252). London, England: Hogarth Press. (Original work published 1919.)

Gabbard, G. O., and Twemlow, S. W. (1991). Do near-death experiences occur only near death-revisited. Journal of Near-Death Studies, 10, 41-47.

Gallup, G. H., Jr, and Newport, F. (1991). Belief in paranormal phenomena among adult Americans. Skeptical Inquirer, 15, 137-146.

Ganaway, G. (1989). Historical truth versus narrative truth: Clarifying the role of exogenous trauma in the etiology of multiple personality disorder and its variants. Dissociation, 2, 205-220.

Globus, G. (1973a). Consciousness and brain: I. The identity thesis. Archives of General Psychiatry, 29, 153-160.

Globus, G. (1973b). Consciousness and brain: II. Introspection, the qualia of experience, and the unconscious. Archives of General Psychiatry, 29, 167-176.

Globus, G., and Franklin, S. (1980). Prospects for the scientific observer of perceptual consciousness. In J. Davidson and R. Davidson (Eds.), The psychobiology of consciousness (pp. 465-481). New York, NY: Plenum.

Hopkins, B. (1981). Missing time: A documented study of UFO abductions. New York, NY: Marek.

Hopkins, B., Jacobs, D. M., and Westrum, R. (1992). Unusual personal experiences: An analysis of the data from three national surveys. Las Vegas, NV: Bigelow Holding Corporation.

Irwin, H. J., and Bramwell, B. A. (1988). The devil in heaven: A near-death experience with both positive and negative facets. Journal of Near-Death Studies, 7, 38-43.

Jacobs, D. M. (1992). Secret life: Firsthand accounts of UFO abductions. New York, NY: Simon and Schuster.

Jones, F. C., Gabbard, G. O., and Twemlow, S. W. (1982). Psychological and demographic characteristics of persons reporting out-of-body experiences. Hillside Journal of Clinical Psychiatry, 6, 105-115.

Kris, E. (1975). Psychoanalysis and the study of creative imagination. In Selected Papers of Ernst Kris (pp. 473-493). New Haven, CT: Yale University Press.

Lane, D. C. (1984). The Himalayan connection: UFOs and the Chandian effect. Journal of Humanistic Psychology, 24, 75-89.

Laibow, R. (1989, May/June). Dual victims: The abused and the abducted. International UFO Reporter, 14(3), 4-9.

McGorry, P. D., Chanen, A., McCarthy, E., Van Riel, R., McKenzie, D., and Singh, B. S. (1991). Post-traumatic stress disorder following recent onset psychosis. Journal of Nervous and Mental Disease, 179, 253-258.

Polanyi, M. (1959). The study of man. Chicago, IL: University of Chicago Press.

Powers, S. M. (1991). Fantasy-proneness, amnesia, and the UFO abduction phenomenon. Dissociation, 4(1), 46-54.

Ramzy, I. (1956). From Aristotle to Freud: A few notes on the roots of psychoanalysis. Bulletin of the Menninger Clinic, 20, 112-123. 
Rahula, W. (1974). What Buddha taught. New York, NY: Grove Press.

Ring, K. (1989). Toward an imaginal interpretation of UFO abductions. ReVision, 11(4), 17-24.

Ring, K., and Rosing, C. (1990). The Omega Project: An empirical study of the NDEprone personality. Journal of Near-Death Studies, 8, 211-239.

Rosenthal, R. (1977). Biasing effects of experimenters. Et cetera, 34, 253-264.

Shacklett, R. L., and Gough, W. C. (1991). The unification of mind and matter. Los Altos, CA: Foundation for Mind-Being Research.

Stern, D. N. (1985). The interpersonal world of the infant: A view from psychoanalysis and developmental psychology. New York, NY: Basic Books.

Tart, C. T. (1972). States of consciousness and state-specific sciences. Science, 176, 12031210.

Twemlow, S. W. (1989). Clinical approaches to the out-of-body experience. Journal of Near-Death Studies, 7, 29-42.

Twemlow, S. W., Gabbard, G. O., and Jones, F. C. (1982). The out-of-body experience: A phenomenological typology based on questionnaire responses. American Journal of Psychiatry, 139, 450-455.

Twemlow, S. W., Hendren, R., Gabbard, G., Jones, F., and Norris, P. (1982). Egointegrating function of psi states. Journal of Psychiatric Treatment and Evaluation, 4, $41-49$.

Wilber, K. (1981). Ontogenetic development: Two fundamental patterns. Journal of Transpersonal Psychology, 13, 33-58.

Wilson, S. C., and Barber, T. X. (1983). The fantasy-prone personality: Implications for understanding imagery, hypnosis, and parapsychological phenomena. In A. A. Sheikh (Ed.), Imagery: Current theory, research, and application (pp. 340-390). New York, NY: Wiley.

Yankelovitch, D., and Barrett, W. (1970). Ego and instinct. New York, NY: Random House. 